\title{
Heating of quasiparticles driven by oscillations of the order parameter in short superconducting microbridges
}

\author{
D. Y. Vodolazov ${ }^{1, *}$ and F. M. Peeters ${ }^{2}$ \\ ${ }^{1}$ Institute for Physics of Microstructures, Russian Academy of Sciences, 603950 Nizhny Novgorod, GSP-105, Russia \\ ${ }^{2}$ Departement Fysica, Universiteit Antwerpen, Groenenborgerlaan 171, B-2020 Antwerpen, Belgium
}

(Received 8 February 2011; published 29 June 2011)

\begin{abstract}
We predict "heating" of quasiparticles driven by order parameter oscillations in the resistive state of short superconducting microbridges. The finite relaxation time of the magnitude of the order parameter $|\Delta|$ and the dependence of the spectral functions both on $|\Delta|$ and the supervelocity $Q$ are the origin of this effect. Our results are opposite to those of Aslamazov and Larkin [Zh. Eks. Teor. Fiz. 70, 1340 (1976)] and Schmid et al. [Phys. Rev. B 215076 (1980)] where “cooling” of quasiparticles was found.
\end{abstract}

DOI: 10.1103/PhysRevB.83.224523

PACS number(s): 74.78.Na, 73.23.-b, 74.20.De, 74.25.Op

\section{INTRODUCTION}

We study theoretically the resistive state in short superconducting microbridges [with length $L$ less than the coherence length $\xi(T)]$ at temperatures close to the critical one. This subject was widely discussed and studied in the 1970s of last century (for review, see Ref. 1) and has regained renewed interest recently (see, for example, Refs. 2 and 3). Interesting phenomena occurring in such a system, and which are still not completely understood, are: (i) the hysteresis of currentvoltage (IV) characteristics at relatively low temperatures ${ }^{1}$ and (ii) the "foot"-like (sometimes also called "shoulder"like, see Ref. 4) feature in the IV characteristics observed experimentally mainly in tin microbridges at low voltages and at temperatures close to $T_{c}$ (Refs. 5-7) (see also Ref. 1). The hysteresis is usually explained by Joule heating while for the "foot"-like structure several theories were proposed ${ }^{8-10}$ that are based on the idea that the quasiparticle distribution function is out of equilibrium (overcooled) as a consequence of the variation in time of the magnitude of the superconducting order parameter $\Delta=|\Delta| e^{i \phi}$ in the superconducting microbridge. The motivation for those theories comes from the fact that the energy of the quasiparticles depends on $|\Delta|$ and when the characteristic time scale for the variation of $|\Delta|$ is smaller than the inelastic relaxation time $\tau_{i n}$ of the quasiparticle distribution function $f(\epsilon)$, the occupancy of the states with energy $\epsilon$ may differ from the equilibrium one (for a detailed discussion, see the book of Tinkham ${ }^{11}$ ).

To simplify the analytical treatment of the problem, the authors of Refs. 8 and 9 assumed that in the dynamic (resistive) state, $|\Delta|$ varies as fast as $\delta \phi$ and to find the coordinate and time dependence of $|\Delta|(x, t)$, they solved the stationary GinzburgLandau equation with a time dependent $\delta \phi(t)$. Furthermore, they assumed that: (1) the relaxation term in the kinetic equations can be neglected when the period of oscillations of the order parameter is much smaller than $\tau_{i n}$, (2) the spectral functions depend only on the local magnitude of the order parameter $|\Delta|$ (so called local approach). We argue that for a realistic inelastic relaxation time $\tau_{i n}$, and even for a short microbridge $L \lesssim \xi(T)$, the nonequilibrium contributions to $f(\epsilon)$ strongly affect the dynamics of $|\Delta|$ and results in a larger time scale for $|\Delta|$ than for $\delta \phi$. Moreover, the relaxation term in the kinetic equations plays a very important role at any voltage, and therefore, cannot be omitted. Taken together with the dependence of the spectral functions on both $|\Delta|$ and $Q$, they provide an averaged "heating" of the quasiparticles, instead of "cooling". 8,9 Some kind of "cooling" of quasiparticles at high voltages can be found only when one takes into account the additional terms in the kinetic equations which couple the longitudinal $f_{L}$ (odd in energy) and transverse $f_{T}$ (even in energy) parts of $2 f(\epsilon)=\left(1-f_{L}(\epsilon)-f_{T}(\epsilon)\right)$ due to the finite spectral supercurrent (in previous works, ${ }^{8,9}$ these terms were omitted). This "cooling" is not effective at low voltages and "heating" together with the large time scale of the variation of $|\Delta|$ results in a hysteresis of the IV characteristics for relatively large $\tau_{i n}$. We should stress that the time averaged "heating" of quasiparticles is driven by oscillations of $|\Delta|$ in the superconducting microbridge and not by Joule dissipation $\sim \mathbf{j} \cdot \mathbf{E}$ (j is the current density and $\mathbf{E}$ is the electric field).

The paper is organized as follows. In Sec. II, we discuss the theoretical model. In Sec. III, we present and discuss our results. In Sec. IV, we present our conclusions and discuss the possible origin of the experimentally found ${ }^{5-7}$ "foot"-like structure in the IV characteristics.

\section{MODEL}

To simulate the resistive state in a short superconducting microbridge, we use the kinetic equations derived in Refs. $12-15$ for "dirty" superconductors near $T_{c}$ :

$$
\begin{gathered}
N_{1} \frac{\partial \delta f_{L}}{\partial t}=D \nabla\left(\left(N_{1}^{2}-R_{2}^{2}\right) \nabla \delta f_{L}\right)+D \nabla\left(j_{\epsilon} f_{T}\right) \\
-\frac{N_{1}}{\tau_{i n}} \delta f_{L}-R_{2} \frac{\partial f_{L}^{0}}{\partial \epsilon} \frac{\partial|\Delta|}{\partial t}, \\
\frac{\partial}{\partial t} N_{1}\left(f_{T}+e \varphi \frac{\partial f_{L}^{0}}{\partial \epsilon}\right) \\
=D \nabla\left(\left(N_{1}^{2}+N_{2}^{2}\right) \nabla f_{T}\right)+D \nabla\left(j_{\epsilon} \delta f_{L}\right) \\
-\frac{N_{1}}{\tau_{i n}}\left(f_{T}+e \varphi \frac{\partial f_{L}^{0}}{\partial \epsilon}\right)-N_{2}|\Delta|\left(2 f_{T}-\frac{\partial f_{L}^{0}}{\partial \epsilon} \frac{\partial \phi}{\partial t}\right),
\end{gathered}
$$

here $Q=(\partial \phi / \partial x-2 e A / c)$ is a quantity which is proportional to the superfluid velocity $\left(v_{s}=D Q\right), \varphi$ is an electrostatic potential, $\delta f_{L}=f_{L}-f_{L}^{0}$ and $f_{L}^{0}(\epsilon)=\tanh \left(\epsilon / 2 k_{B} T\right)$. $N_{1}, N_{2}, R_{2}$ are the spectral functions which should be 
found from the Usadel equation for the normal $\alpha(\epsilon)=$ $\cos \Theta=N_{1}(\epsilon)+i R_{1}(\epsilon)$ and anomalous $\beta_{1}=\beta e^{i \phi}, \beta_{2}=$ $\beta e^{-i \phi}\left(\beta(\epsilon)=\sin \Theta=N_{2}(\epsilon)+i R_{2}(\epsilon)\right)$ Green functions

$$
\begin{aligned}
& \hbar D \frac{d^{2} \Theta}{d x^{2}}+\left(\left(2 i \epsilon-\frac{\hbar}{\tau_{i n}}\right)-\hbar D Q^{2} \cos \Theta\right) \sin \Theta \\
& \quad+2|\Delta| \cos \Theta=0
\end{aligned}
$$

Equations (1a) and (1b) are coupled through the finite spectral supercurrent ${ }^{13,16} \quad j_{\epsilon}=\operatorname{Re}\left(\beta_{1} \nabla \beta_{2}-\beta_{2} \nabla \beta_{1}\right) / 2=$ $2 N_{2} R_{2} Q$. Below we show that the coupling terms in Eqs. (1a) and (1b) strongly influence $\delta f_{L}$ and the dynamics of the order parameter which is described by the modified time-dependent Ginzburg-Landau equation

$$
\begin{aligned}
& \frac{\pi \hbar}{8 k_{B} T_{c}} \frac{\partial \Delta}{\partial t}+\left(\Phi_{1}+i \Phi_{2}\right) \Delta \\
& \quad=\xi_{G L}^{2} \frac{\partial^{2} \Delta}{\partial x^{2}}+\left(1-\frac{T}{T_{c}}-\frac{|\Delta|^{2}}{\Delta_{G L}^{2}}\right) \Delta,
\end{aligned}
$$

where $\xi_{G L}^{2}=\pi \hbar D / 8 k_{B} T_{c}$ and $\Delta_{G L}^{2}=8 \pi^{2}\left(k_{B} T_{c}\right)^{2} / 7 \zeta(3)$ are the zero temperature Ginzburg-Landau coherence length and the corresponding order parameter. Nonequilibrium parts of the quasiparticle distribution function enter Eq. (3) via the potentials $\Phi_{1}=-\int_{0}^{\infty} R_{2} \delta f_{L} d \epsilon /|\Delta|$ and $\Phi_{2}=$ $-\int_{0}^{\infty} N_{2} f_{T} d \epsilon /|\Delta|$. When $\delta f_{L}$ is negative, the potential $\Phi_{1}$ is positive and vice versa. In some respect, from the structure of Eq. (3), it follows that one may introduce an "effective" temperature for the quasiparticles $T_{\mathrm{eff}}(x, t)=T+\Phi_{1}(x, t) T_{c}$ and thus the positive/negative sign of $\Phi_{1}(x, t)$ means local "heating"/"cooling" of quasiparticles. We should stress that we use the term "effective" temperature only in order to give a simple physical interpretation of our numerical results and describe the integral effect of the nonequilibrium distribution $f(\epsilon)$ which enters the equation for the order parameter via the potentials $\Phi_{1}(x, t)$ and $\Phi_{2}(x, t)$. Please note that the resulting nonequilibrium $f(\epsilon)$ cannot be viewed as a Fermi-Dirac function with effective temperature $T_{\text {eff }}(x, t)$ and electrostatic potential $\varphi(x, t)$.

The current and the electrostatic potential in the sample can be found using the following equations:

$$
\begin{gathered}
j=\frac{\sigma_{n}}{e}\left(\frac{|\Delta|^{2} Q}{4 k_{B} T_{c}}+\int_{0}^{\infty}\left(\left(N_{1}^{2}+N_{2}^{2}\right) \nabla f_{T}+j_{\epsilon} \delta f_{L}\right) d \epsilon\right), \\
e \varphi=-\int_{0}^{\infty} N_{1} f_{T} d \epsilon / \int_{0}^{\infty} N_{1} \frac{\partial f_{L}^{0}}{\partial \epsilon} d \epsilon
\end{gathered}
$$

where $\sigma_{n}$ is the normal state conductivity. In metals, we have for the charge density, $\rho \simeq 0$ and the condition $d i v j=0$ is satisfied due to Eqs. (1b), (3), and (5).

In the derivation of Eqs. (1a) and (1b), it was assumed that deviations from the equilibrium are small $\delta f_{L}, f_{T} \ll f_{L}^{0}$. It allowed one to linearize the collision integral due to electron-phonon collisions and to write it in the relaxation time approximation. Furthermore, it was assumed that the inelastic relaxation time due to electron-electron interactions is much

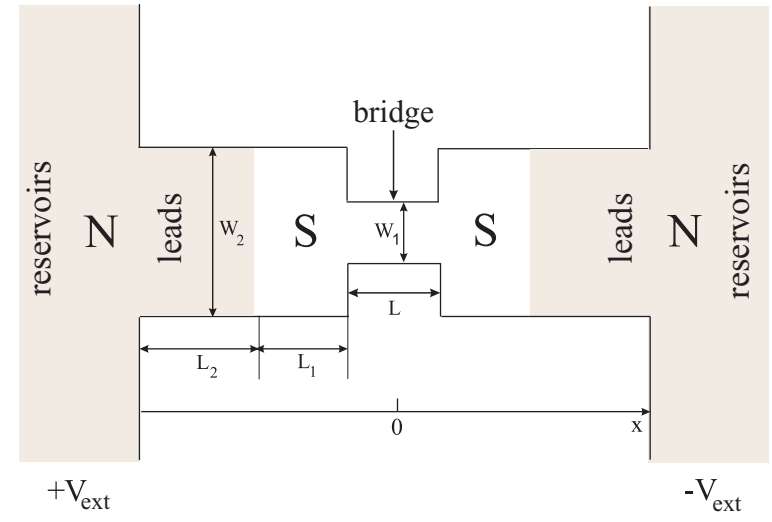

FIG. 1. (Color online) Schematic illustration of the model system with the geometric parameters.

larger than due to electron-phonon and hence one can neglect the corresponding collision integral.

To model the superconducting microbridge connected to superconducting bulk leads in the current carrying regime, we consider the system shown in Fig. 1. It consists of normal bulk reservoirs where the external voltage $V_{\text {ext }}$ is applied and the constriction is modeled as a film with variable cross section $S_{2} \gg S_{1}$ (here $S_{1(2)}=W_{1(2)} d_{1(2)}$ is the cross section, $W_{1(2)}$ is the width, and $d_{1,2}$ is the thickness of the film (in this way, we model variable thickness microbridge). The narrowest part of the film is called the microbridge and the wide parts are assigned as being the leads. The wide part of the film of length $2 L_{2}$ is in the normal state and the rest of the film is in the superconducting state (for relatively small $V_{\text {ext }}$ ). In our calculations, we chose $L_{2} / L_{1} \gg 1$ and the length $L_{1}$ was taken large enough to neglect nonequilibrium effects from the NS boundaries located at $x= \pm\left(L_{1}+L / 2\right)$ on the transport properties of the microbridge. The large normal part of the film is needed because the voltage $V$ enters in the boundary conditions for $f_{L}$ and $f_{T}$ (see, for example, Ref. 16) and almost all the voltage drop occurs in the normal part of the film, effectively leading to a state with applied constant current even when the microbridge transits to the resistive state. Due to the large ratio $S_{2} / S_{1} \gg 1$, the transport current does not destroy superconductivity in the wide part of the film and we mainly study the resistive state in the microbridge. In our calculations, we assume that the lateral size of the film is much smaller than the London penetration depth $\lambda$ (or effective penetration depth $\left.\lambda_{\perp}=\lambda^{2} / d_{1(2)}\right)$, and therefore, we may neglect screening effects. Furthermore, we suppose that the current density is distributed uniformly over the width of the film. This assumption is, strictly speaking, not valid in the region where the current transits from the wide part to the narrow one (and vice versa). But because the current density is much smaller in the wide part than in the narrow part, we may neglect such type of effects (at least for variable thickness microbridges) and consider variables which are averaged over the width and thickness of the film. Because the length of the microbridge is much larger than its width, we may assume a uniform current distribution over the width of the microbridge. Thus, our system is quasi-1D and we should only take into account the continuity of $\Delta, \Theta, f_{T}, \delta f_{L}$, and conservation of the superconducting [Eq. (6a) below], normal [Eq. (6d)], 
energy [Eq. (6c)], and spectral [Eq. (6b)] currents at the points $x= \pm L / 2$ through the boundary conditions

$$
\begin{gathered}
S_{2} \partial \Delta /\left.\partial x\right|_{ \pm L / 2}=S_{1} \partial \Delta /\left.\partial x\right|_{ \pm L / 2}, \\
S_{2} \partial \Theta /\left.\partial x\right|_{ \pm L / 2}=S_{1} \partial \Theta /\left.\partial x\right|_{ \pm L / 2}, \\
\left.S_{2} D\left(\left(N_{1}^{2}-R_{2}^{2}\right) \partial \delta f_{L} / \partial x+j_{\epsilon} f_{T}\right)\right|_{ \pm L / 2} \\
\left.=S_{1} D\left(N_{1}^{2}-R_{2}^{2}\right) \partial \delta f_{L} / \partial x+j_{\epsilon} f_{T}\right)\left.\right|_{ \pm L / 2}, \\
\left.S_{2} D\left(\left(N_{1}^{2}+N_{2}^{2}\right) \partial f_{T} / \partial x+j_{\epsilon} \delta f_{L}\right)\right|_{ \pm L / 2} \\
=\left.S_{1} D\left(\left(N_{1}^{2}+R_{2}^{2}\right) \partial f_{T} / \partial x+j_{\epsilon} \delta f_{L}\right)\right|_{ \pm L / 2} .
\end{gathered}
$$

It is easy to find the solution to Eqs. (1)-(5) in the normal region, and therefore, we only need to solve them in the superconducting region (in the interval, $-L_{1}-L / 2<x<L_{1}+$ $L / 2)$ with the following boundary conditions: $\left.\Delta\right|_{ \pm\left(L_{1}+L / 2\right)}=$ $0,\left.\Theta\right|_{ \pm\left(L_{1}+L / 2\right)}=0,\left.\delta f_{L}\right|_{ \pm\left(L_{1}+L / 2\right)}=0,\left.f_{T}\right|_{ \pm\left(L_{1}+L / 2\right)}=-e \varphi_{ \pm}$. $\partial f_{L}^{0} / \partial E$, where $\varphi_{ \pm}=\mp V_{\text {ext }} \pm j_{ \pm} L_{2} / \sigma_{n}$ and the current density $j_{ \pm}$in the points $x= \pm\left(L_{1}+L / 2\right)$ could be found from Eq. (4).

In our numerical calculations, we use dimensionless units. The order parameter is scaled by $\Delta_{0}\left(\Delta_{0}=1.76 k_{B} T_{c} \simeq\right.$ $0.57 \Delta_{G L}$ is the zero temperature order parameter value in the weak coupling limit), distance is in the units of the zero temperature coherence length $\xi_{0}=\sqrt{\hbar D / \Delta_{0}} \simeq 1.2 \xi_{G L}$, time is in the units of $t_{0}=\hbar / \Delta_{0}$, and temperature in the units of the critical temperature $T_{c}$. The current is scaled in the units of $j_{0}=\Delta_{0} \sigma_{n} /\left(\xi_{0} e\right)$, the superfluid velocity is in the units of $Q_{0}=\hbar c / 2 e \xi_{0}$, and the electrostatic potential is in the units of $\varphi_{0}=\Delta_{0} / e$. It is useful to introduce the dimensionless inelastic relaxation time $\tilde{\tau}_{\text {in }}=\tau_{\text {in }} / t_{0}$ which is the main control parameter in the model described by Eqs. (1a) and (1b).

We used the implicit method for the numerical solution of Eqs. (1)-(3). The coordinate step of the grid was equal to $\xi_{0}$ (which is much smaller than $\xi(T)$ for the considered temperature interval $0.92<T / T_{c}<0.99$ ) and the time step varied from $0.5 t_{0}$ up to $2 t_{0}$ depending on the temperature. In our numerical procedure, we use an even number of grid points and consequently the center of the microbridge where the order parameter goes to zero (the phase slip center) is situated between grid points. Therefore, any quantity in the phase slip center (PSC) is in fact calculated at a distance $\delta x=$ $\xi_{0} / 2 \ll \xi(T)$ from the PSC.

For the geometrical parameters of the film, we used the following values: $S_{2} / S_{1}=10, L_{2}=500 \xi_{0}$, and $L_{1}$ was varied from $15 \xi_{0}$ (at $T=0.92 T_{c}$ ) up to $45 \xi_{0}$ (at $T=0.99 T_{c}$ ). In our calculations, we used $\tilde{\tau}_{\text {in }}=4-1000$ which covers typical values for many low temperature superconductors (for example, Ref. 15 in $\mathrm{Nb} \tilde{\tau}_{\text {in }} \simeq 10^{2}$ and in $\mathrm{Al} \tilde{\tau}_{\text {in }} \simeq 10^{3}$ ). In the wide part of the film, we used $\tilde{\tau}_{\text {in }}=5$ which allows us to decrease $L_{i n}$ and the length $L_{1}$ in order to optimize the calculation time.

To find the current-voltage characteristics of the superconducting microbridge we applied a large voltage $\pm V_{\text {ext }}$ to the normal reservoirs which induces a large current $\left(I>I_{c}\right)$ resulting in a resistive state in the microbridge. Then we decrease $V_{\text {ext }}$ in a step-wise manner and we find the time averaged difference of the electrostatic potentials between the ends of the microbridge $(\bar{V}=\bar{\varphi}(-L / 2)-\bar{\varphi}(L / 2))$ as a function of $V_{\text {ext }}$. In a similar way, it is easy to find the critical voltage $V_{\text {ext }}^{c}$ (or critical current $I_{c}$ ) of the superconducting

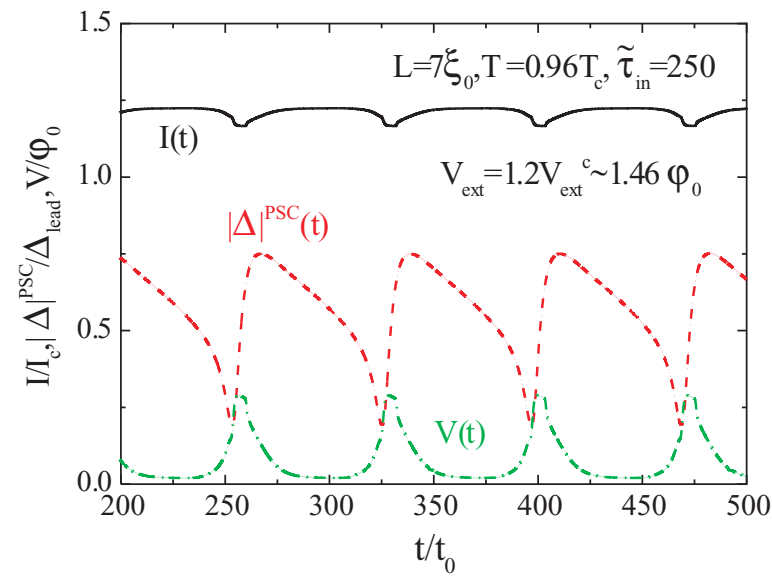

FIG. 2. (Color online) Time dependence of the current, amplitude of the order parameter in the center of the microbridge and voltage drop over the microbridge at fixed external voltage $V_{\text {ext }}$. The results were found from numerical solution of Eqs. (1)-(5) in the presence of coupling terms in Eqs. (1a) and (1b). The geometrical parameters of the system are: $W_{2} / W_{1}=10, L_{2}=500 \xi_{0}, L_{1}=22 \xi_{0}$, and $L=$ $7 \xi_{0}$.

microbridge when the superconducting state becomes unstable (by increasing $V_{\text {ext }}$ from small values). As a result, we find the dependence $\bar{V}\left(V_{\text {ext }} / V_{\text {ext }}^{c}\right)$ which practically coincides with the dependence $\bar{V}\left(I / I_{c}\right)$. In Fig. 2, we plot the time dependence of the current in the microbridge which illustrates that indeed $I$ varies weakly in time and the constant $V_{\text {ext }}$ induces an almost constant $I$ in our model system even when the microbridge is in the resistive state.

\section{RESULTS}

In Fig. 3, we show the current-voltage characteristics of a short microbridge with length $L=7 \xi_{0}$ at $T=0.96 T_{c}$ calculated for different values of the inelastic relaxation time $\tau_{i n}$ in the regime of decreasing applied current (the length and temperature were chosen close to the parameters of the experiment of Ref. 7 and for tin $\tilde{\tau}_{i n} \sim 200$ ). First, we should note the strong influence of the coupling terms in Eqs. (1a) and (1b) on the IV characteristics: voltage increases (decreases) with increasing $\tau_{i n}$ at $I \gtrsim I_{c}$ in case of the absence (presence) of coupling terms. Second, in both cases, the IV curves are hyperbolic-like with no "foot"-like feature and with hysteresis for relatively large $\tau_{i n}$ (the transition to the superconducting state occurs at the retrapping current $I_{r}$ which could be smaller than the critical current $I_{c}$ for the transition from the superconducting to the resistive state).

We plot in Fig. 4, the energy dependence of $\delta f_{L}$ averaged over one oscillation period $T_{|\Delta|}$ taken in the phase slip center. The effect of the coupling terms are clearly visible by comparing Figs. 4(a) and 4(b). The $\overline{\delta f}_{L}^{\text {PSC }}$ is on the average negative in case the coupling terms are absent and positive when they are included.

Let us now discuss the origin of the sign of $\overline{\delta f}_{L}^{\mathrm{PSC}}$ for the different energies. First, we consider the case when the 


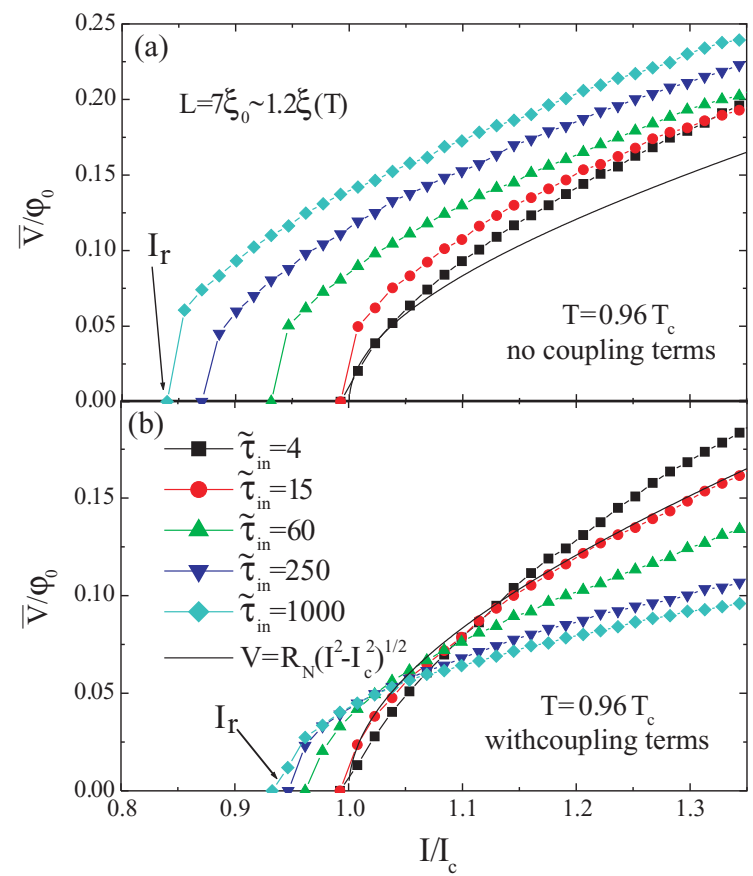

FIG. 3. (Color online) Current-voltage characteristics (in the regime of decreasing current) for a short microbridge with length $L=7 \xi_{0}$ at $T=0.96 T_{c}$ calculated for different values of the inelastic relaxation time in the absence (a) and the presence (b) of coupling terms in Eqs. (1a) and (1b). Solid curve is the IV characteristic of a short microbridge in the absence of nonequilibrium effects (see, for example, Ref. 4).

coupling terms are neglected. Let us simplify Eq. (1a) to

$$
\frac{\partial \delta f_{L}}{\partial t}=-\frac{\delta f_{L}}{\tau_{i n}}-\frac{R_{2}}{N_{1}} \frac{\partial f_{L}^{0}}{\partial \epsilon} \frac{\partial|\Delta|}{\partial t},
$$

where we omit the diffusive and coupling terms. If we average Eq. (7) over $T_{|\Delta|}$ then we obtain

$$
\overline{\delta f}_{L}=-\tau_{i n} \frac{\partial f_{L}^{0}}{\partial \epsilon} \int_{0}^{T_{|\Delta|}} \frac{R_{2}}{N_{1}} \frac{\partial|\Delta|}{\partial t} d t .
$$

If $R_{2}$ and $N_{1}$ are solely a function of $|\Delta|$ then $\overline{\delta f}_{L}=0$. But in general the spectral functions $R_{2}$ and $N_{1}$ are a function of two variables $|\Delta|,|Q|$ [at fixed $x$ and $\epsilon$, see Eq. (2)]. To get the insight that how the ratio $R_{2} / N_{1}$ changes with varying $|Q|$ at fixed $|\Delta|$, one may solve Eq. (2) with zero second derivative and find that the ratio $R_{2} / N_{1}$ decreases when $\epsilon \gtrsim|\Delta|$ and increases when $\epsilon \lesssim|\Delta|$ for large $|Q|$.

In Fig. 5, we present the time dependence of $|\Delta|, Q$, and $\Phi_{1}$ in the absence of the coupling terms in Eqs. (1a) and (1b). First of all, we should note that the amplitude of the oscillations of $|\Delta|$ is smaller than $\Delta_{\text {lead }}$. The reason for this effect is the large characteristic time scale for the variation of $|\Delta|$ in comparison with the one of $\delta \phi$. Indeed it is known that nonequilibrium effects may considerably slow down the dynamics of $|\Delta| .^{17-19}$ When $|\Delta|$ increases (decreases), its value is smaller (larger) than one could expect from the static dependence $|\Delta|_{\text {stat }}(\delta \phi)$ (see, for example, Eq. (1.3) in Ref. 4) for the given value of $\delta \phi(t) \sim Q(t) L$. For example, when $\delta \phi$ reaches zero (which corresponds to the moment in time when $Q=0$ after the phase

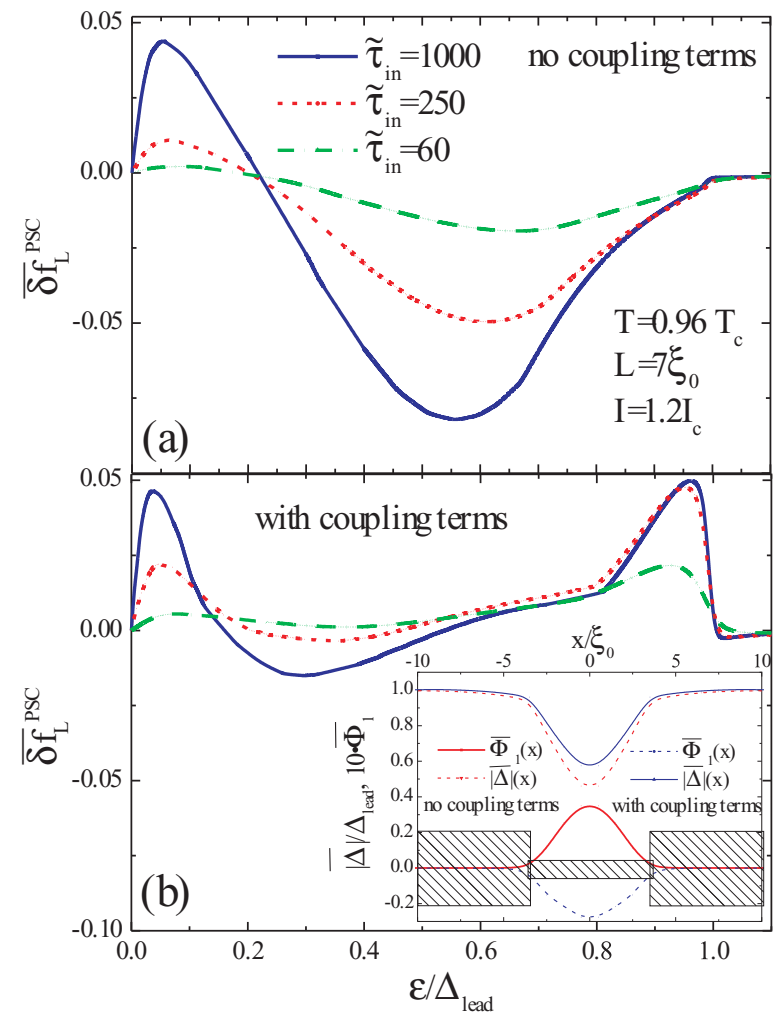

FIG. 4. (Color online) Typical energy dependence of the time averaged $\delta f_{L}$ in the center of the short microbridge (i.e., in the phase slip center) in the absence (a) and the presence (b) of the coupling terms in Eqs. (1a) and (1b). In the inset of Fig. 4(b), we show the distribution of the time averaged $\Phi_{1}$ and $|\Delta|$ along the microbridge and the leads (schematically shown).

slip event in Fig. 5), the order parameter is still much smaller than $\Delta_{\text {lead }}$.

Due to the time delay in the variation of $|\Delta|$, the supervelocity $Q \sim \delta \phi / L$ is different for the same values of $|\Delta|$ taken

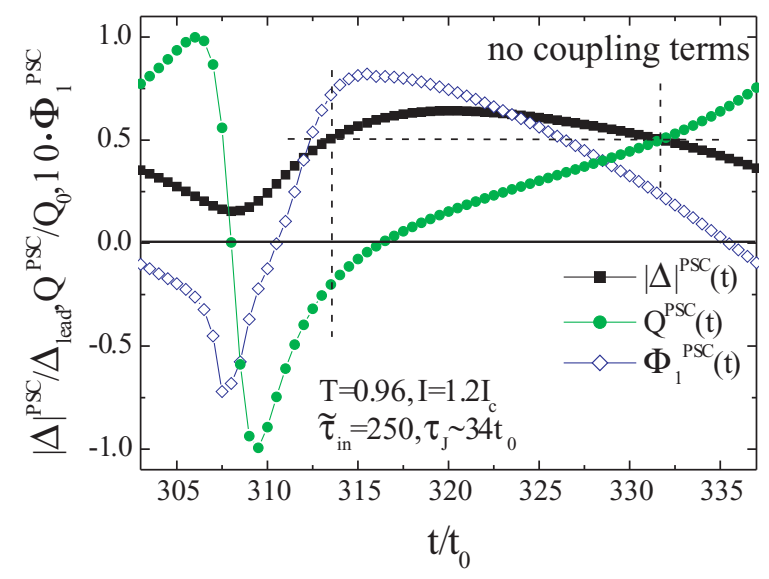

FIG. 5. (Color online) Time dependence of the order parameter $|\Delta|$, the supervelocity $Q$, and the potential $\Phi_{1}$ in the center of the short microbridge (parameters are the same as in Fig. 4) at $I=1.2 I_{c}$ and $\tilde{\tau}_{i n}=250$ as found from a numerical solution of Eqs. (1)-(3) without coupling terms. The horizontal dashed line marks $|\Delta|=\Delta_{\text {lead }} / 2$ to illustrate the corresponding different $Q$ values at two moments in time. 
at different times during the period $T_{|\Delta|}$ (see dashed lines in Fig. 5). Therefore, the ratio $R_{2} / N_{1}$ in the increasing region of $|\Delta|$ is larger (smaller) for $\epsilon \gtrsim|\Delta|(x, t)(\epsilon \lesssim|\Delta|(x, t))$ than in the decreasing region. It results in $\overline{\delta f}_{L}<0$ in the phase slip center for $\epsilon \gtrsim \overline{|\Delta|}^{\mathrm{PSC}}$ and $\overline{\delta f}_{L}>0$ for $\epsilon \lesssim \overline{|\Delta|}^{\mathrm{PSC}}$ [see Fig. 4(a)]. The time averaged potential $\Phi_{1}$ is positive in the microbridge [see inset in Fig. 4(b)] which implies "heating" of quasiparticles and suppression of the superconducting properties. The larger the $\tau_{i n}$ the stronger the deviation form equilibrium [see Eq. (8) and Fig. 4(a)] and the larger the hysteresis [see Fig. (1a)].

Consider now the effect of the coupling terms in Eqs. (1a) and (1b). The term $D \nabla\left(j_{\epsilon} f_{T}\right)=2 D \nabla\left(N_{2} R_{2} Q f_{T}\right)$ in Eq. (1a) may be considered as an additional source of nonequilibrium which is nonzero in the energy interval $\delta \epsilon \simeq Q^{2}$ near local $|\Delta|(x)$ [in the spatially uniform case with $Q=0$ and $\tau_{i n}=$ $\infty$, one has $N_{2} R_{2} \sim|\Delta|(x) \delta(\epsilon-|\Delta|(x))$, see, for example, Ref. 9]. From Eq. (1b), we may roughly estimate $f_{T} \sim$ $-e \varphi \partial f_{L}^{0} / \partial \epsilon$ and the sign of the coupling term $D \nabla\left(j_{\epsilon} f_{T}\right)$ is defined mainly by the product $\mathbf{Q} \cdot \mathbf{E}$ (for discussion of the effect of the coupling terms see also Ref. 19).

Our calculations show (see Fig. 6) that near the superconducting leads, the product $\mathbf{Q} \cdot \mathbf{E}$ is mainly (in time) positive and in the phase slip center, it can be both positive and negative (due to the sign change of $\mathbf{Q}$, see Fig. 5). It results in positive $\overline{\delta f_{L}}$ at $\epsilon \sim \Delta_{\text {lead }}$, see Fig. 4(b) (at these energies, the ends of the microbridge are determinative because near the leads $|\Delta|$ has a low oscillation amplitude and $\overline{\mathbf{Q} \cdot \mathbf{E}}>0$ ). At lower energies $\epsilon \sim \overline{|\Delta|}_{\text {PSC }}<\Delta_{\text {lead }}$, the main source of nonequilibrium is situated in the center of the microbridge where both $\mathbf{Q} \cdot \mathbf{E}$ and $\partial|\Delta| / \partial t$ change sign during the oscillations and $\overline{\delta f_{L}}$ is negative except at low energies $\epsilon \ll \overline{|\Delta|}^{\mathrm{PSC}}$ [see Fig. 4(b)]. The net effect is a negative time averaged $\bar{\Phi}_{1}$ [see inset in Fig. 4(b)], and therefore, an enhancement of the superconducting properties which explains the decrease of the voltage at fixed current $I \gtrsim I_{c}$ with increase of $\tau_{i n}$ [see Fig. 3 (b)]. But at low voltages, the effect of the coupling terms ( QE) becomes smaller and the role of "heating" increases. This subsequently leads to an increase of the voltage at fixed current $I \sim I_{c}$ with increasing $\tau_{i n}$ and to a hysteresis of the IV curves [but which is smaller than in the case without coupling terms, compare Figs. 3(a) and 3(b)].

\section{DISCUSSION}

The origin of the here predicted time averaged "heating" of quasiparticles is different from the Joule dissipation. Indeed, the source of nonequilibrium $\sim \partial|\Delta| / \partial t$ changes sign during oscillations of the order parameter while the term $I \cdot V$ is always positive. Time averaged "heating" appears only due to the dependence of the spectral functions both on $|\Delta|$ and $Q$ and due to the difference in time variation of $|\Delta|$ and $\delta \phi$. Energy for "heating" of the quasiparticles [and "cooling" due to the term $\nabla\left(j_{\epsilon} f_{T}\right)$ in the kinetic equation] comes from the energy $I \cdot V$ delivered by the external source of the current (this energy goes also, for example, to the "heating" of phonons due to the presence of the relaxation term in the kinetic equations).

Our result is opposite to the one of Refs. 8 and 9 where a time averaged "cooling" of quasiparticles and an enhancement

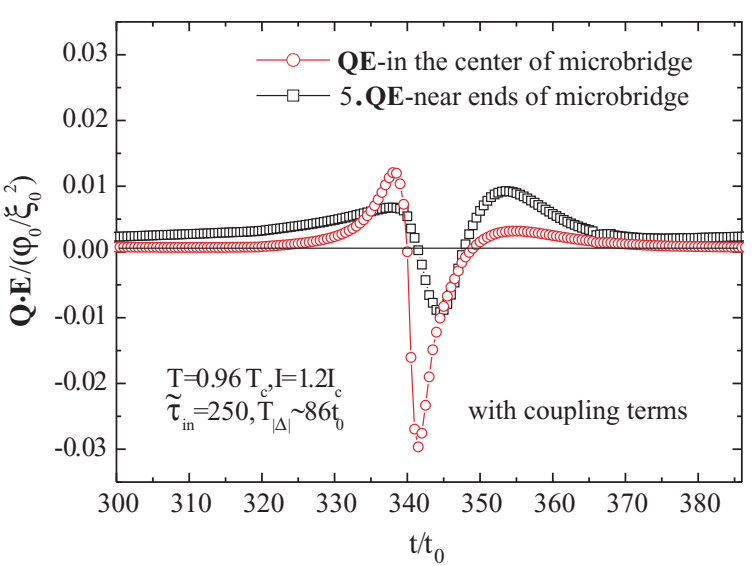

FIG. 6. (Color online) Time dependence of the product $\mathbf{Q} \cdot \mathbf{E}$ in the phase slip center and near the ends of the short superconducting microbridge during $T_{|\Delta|}$ as found from a numerical solution of Eqs. (1)-(3) including the coupling terms.

of superconductivity were found at high voltages (when $\left.T_{|\Delta|} \ll \tau_{i n}\right)$ in the absence of the coupling terms in Eqs. (1a) and (1b). The differences with our calculations are that we take into account: (i) the back action of the nonequilibrium quasiparticle distribution function on the dynamics of $|\Delta|$; and (ii) the dependence of the spectral functions $N_{1}, N_{2}$, and $R_{2}$ on both $|\Delta|$ and $|Q|$. There is also one more difference with Refs. 8 and 9-we did not neglect the relaxation term in Eq. (7) at high voltages [see Eq. (3) in Ref. 8 and Eq. (16) in Ref. 9]. By omitting this term, one may obtain "cooling" with $\overline{\delta f}_{L}>0$ if one chooses the initial condition $\delta f_{L}=0$ when $|\Delta|=\Delta_{\text {lead }}$ in the center of the microbridge (and when the spectral functions $R_{2}$ and $N_{1}$ depend only on $|\Delta|$ as was assumed in Refs. 8 and 9). But this choice of the initial condition is not obvious. By choosing a different initial condition (large negative $\delta f_{L}$ at $|\Delta|=\Delta_{\text {lead }}$ ), one may obtain "heating" in this case too. Only the presence of the relaxation term in Eq. (7) resolves this problem, because it defines undoubtedly the time averaged value of $\delta f_{L}$ [see Eq. (8)]. Note that due to model assumptions for spectral functions of Refs. 8 and $9 \overline{\delta f}_{L}$ has to be equal to zero at any voltage if one does not neglect the relaxation term. From a physical point of view, the relaxation term in Eq. (7) tells us that the accumulation of the "heat"/"cold" (after sudden change of the current, for example) occurs on a time scale which is proportional to $\tau_{i n} \gg T_{|\Delta|}$ and one should take it into account to find the true value of $\overline{\delta f}_{L}$ and $T_{|\Delta|}$ corresponding to the given value of the current. It provides a strong dependence of the IV characteristics and $\overline{\delta f}_{L}$ on $\tau_{\text {in }}$ even at high voltages [see Figs. 3(a) and 4(a)] while in Refs. 8 and 9 no dependence on $\tau_{i n}$ was found in this limit.

According to our numerical calculations the IV characteristics of short "dirty" superconducting microbridges are hyperbolic-like and hysteretic (at relatively large $\tau_{\text {in }}$ and/or low temperatures) in the temperature interval $0.92<T / T_{c}<1$ [calculated and not presented here the IV characteristics at $T / T_{c}=0.92-0.99$ are qualitatively similar to those of Figs. 3(a) and 3(b)]. We believe that the experimentally found "foot"-like features in the IV characteristics of short superconducting microbridges is connected with the geometry of the studied 
microbridges - they were short and wide (in experiments, ${ }^{5-7}$ the width $W \simeq 2 L$ ). For such a geometry, the current density distribution over the width of the microbridge (constriction) should be nonuniform with sharp peaks near the edges of the microbridge. ${ }^{20}$ In Ref. 21 , it was shown that a nonuniform current density distribution in the superconductor may lead to slow vortex motion at low currents and phase slip lines at high currents ${ }^{21}$ and to a IV characteristic (see, for example, Fig. 4 in Ref. 21) which resembles the experimental results of Refs. 5-7. Moreover, the sharp transition from low to high voltages occurs at $V_{c}$ which is inversely proportional to $\tau_{i n}{ }^{21}$ - the same result was found in the experiment on tin superconducting microbridges. ${ }^{6,7}$ Therefore, we predict that the IV characteristics of short $(L \lesssim \xi(T))$ superconducting microbridges should change from hyperbolic-like (in case when $W \ll L)$ to the one with a well-pronounced 'foot'-like structure at low voltages when $W \gtrsim L$. Good candidates are $\mathrm{Sn}, \mathrm{In}, \mathrm{Pb}$, and $\mathrm{Al}$ with relatively large coherence length $\left[\xi(T) \gtrsim 500 \mathrm{~nm}\right.$ at $\left.T \sim T_{c}\right]$. We expect that our results will be tested in the near future because recently it has become possible to fabricate microbridges with length $L<500 \mathrm{~nm}$ and width $W \gtrsim 50 \mathrm{~nm}$ (see, for example, Refs. 2 and 3) which satisfy the condition $W \ll L \lesssim \xi(T)$ at $0.9<T / T_{c}<1$.

\section{ACKNOWLEDGMENTS}

This work was supported by the Russian Foundation for Basic Research, Russian Agency of Education under the Federal Target Programme "Scientific and educational personnel of innovative Russia in 2009-2013," Flemish Science Foundation (FWO-Vl), and the Belgian Science Policy (IAP). *vodolazov@ipm.sci-nnov.ru

${ }^{1}$ K. K. Likharev, Rev. Mod. Phys. 51, 101 (1979).

${ }^{2}$ J. Wang, X. Ma, S. Ji, Y. Qi, Y. Fu, A. Jin, L. Lu, C. Gu, X. C. Xie, M. Tian, J. Jia, and Q. Xue, Nano Res. 2, 671 (2009).

${ }^{3}$ D. Hazra, L. M. A. Pascal, H. Courtois, and A. K. Gupta, Phys. Rev. B 82, 184530 (2010).

${ }^{4}$ L. G. Aslamazov and A. F. Volkov, in Nonequilibrium Superconductivity, edited by D. N. Langenberg and A. I. Larkin (Elsevier, Amsterdam, 1986), Chap. 2.

${ }^{5}$ G. Guthmann, J. Maurer, M. Belin, J. Bok, and A. Libchaber, Phys. Rev. B 11, 1909 (1975).

${ }^{6}$ V. N. Gubankov, V. P. Koshelets, and G. A. Ovsyannikov, Zh. Eksp. Teor. Fiz. 73, 1435 (1977) [Sov. Phys. JETP 46, 755 (1977)].

${ }^{7}$ M. Octavio, W. J. Skocpol, and M. Tinkham, Phys. Rev. B 17, 159 (1978).

${ }^{8}$ L. G. Aslamazov and A. I. Larkin, Zh. Eksp. Teor. Fiz. 70, 1340 (1976) [Sov. Phys. JETP 43, 698 (1976)].

${ }^{9}$ A. Schmid, G. Schön, and M. Tinkham, Phys. Rev. B 21, 5076 (1980).

${ }^{10}$ A. A. Golub, Zh. Eksp. Teor. Fiz. 71, 341 (1976) [Sov. Phys. JETP 44, 178 (1976)].
${ }^{11}$ M. Tinkham, Introduction to Superconductivity (McGraw-Hill, NY, 1996), p. 417.

${ }^{12}$ A. Schmid and G. Schön, J. Low Temp. Phys. 20, 207 (1975).

${ }^{13}$ A. I. Larkin and Yu. N. Ovchinnikov, Zh. Eksp. Teor. Fiz. 73, 299 (1977) [Sov. Phys. JETP 46, 155 (1977)].

${ }^{14}$ L. Kramer and R. J. Watts-Tobin, Phys. Rev. Lett. 40, 1041 (1978).

${ }^{15}$ R. J. Watts-Tobin, Y. Krähenbühl, and L. Kramer, J. Low Temp. Phys. 42, 459 (1981).

${ }^{16}$ R. S. Keizer, M. G. Flokstra, J. Aarts, and T. M. Klapwijk, Phys. Rev. Lett. 96, 147002 (2006).

${ }^{17}$ J. A. Pals and J. Wolter, Phys. Lett. A 70, 150 (1979) (see also Ref. 11, p. 414).

${ }^{18} \mathrm{M}$. Tinkham, in Non-Equilibrium Superconductivity, Phonons and Kapitza Boundaries, edited by K. E. Gray (Plenum, New York, 1981).

${ }^{19}$ D. Yu. Vodolazov and F. M. Peeters, Phys. Rev. B 81, 184521 (2010).

${ }^{20}$ L. G. Aslamazov and A. I. Larkin, Zh. Eksp. Teor. Fiz. 68, 766 (1975) [Sov. Phys. JETP 41, 381 (1975)].

${ }^{21}$ D. Yu. Vodolazov and F. M. Peeters, Phys. Rev. B 76, 014521 (2007). 